\title{
Psicoterapia para Crianças e Adolescentes Vítimas de Violência Sexual no Sistema Público: Panorama e Alternativas de Atendimento
}

Psychotherapy for Children and Adolescents Victims of Sexual Violence in the Brazilian Public Health System:

Overview and Treatment Alternatives

Psicoterapia para Niños y Adolescentes Víctimas de Violencia Sexual en el Sistema Público Brasileño: Panorama y Alternativas de Tratamiento

Jean Von Hohendorff \& Silvia Helena Koller

Universidade Federal do Rio Grande do Sul

Luísa Fernanda Habigzang Pontifícia Universidade Católica do Rio Grande do Sul 
Resumo: O objetivo deste ensaio é abordar a questão da garantia de acesso ao tratamento psicoterápico de crianças e adolescentes vítimas de violência sexual pelo sistema público, tendo em vista que o desenvolvimento de psicopatologias em curto e em longo prazo está associado à experiência de violência sexual. As principais leis e diretrizes sobre a organização dos serviços públicos de assistência social e de saúde para vítimas de violência sexual foram revisadas, bem como estudos sobre os encaminhamentos adotados nesses casos. Constatou-se que o serviço público de assistência deve oferecer atendimento psicossocial, enquanto o serviço público de saúde deve oferecer psicoterapia. No entanto, os estudos sobre encaminhamentos de casos de violência sexual contra crianças e adolescentes indicam baixos índices de encaminhamento para serviços que oferecem psicoterapia. Tal panorama é discutido a partir das diretrizes quanto ao público atendido pelo serviço público de saúde no que tange à saúde mental, bem como a partir da quantidade desses serviços em nosso país. Alternativas para a garantia de acesso ao tratamento psicoterápico de crianças e adolescentes vítimas de violência sexual pelo sistema público de saúde são discutidas.

Palavras-chave: Violência sexual. Criança. Adolescente. Psicoterapia.

Abstract: This article aims to address the issue of ensuring access to psychotherapeutic treatment to children and adolescents victims of sexual violence in the public health system in view of the association between exposure to sexual violence and the development of short and long term psychopathologies. The main laws and guidelines in the organization of the Brazilian public services of social assistance and health care for victims of sexual violence were reviewed, as well as studies on referrals conducted in these cases. It was found that the social assistance public service should offer psychosocial treatment, while the health care public service should offer psychotherapy. However, studies addressing cases of sexual violence against children and adolescents indicate low rates of referrals to services that offer psychotherapy. This scenario is discussed based on the guidelines regarding the population assisted in the mental health public service, as well as on the figures of these services in Brazil. Alternatives to ensure access to psychotherapeutic treatment of children and adolescents victims of sexual violence by the public health system are discussed.

Keywords: Sexual violence. Children. Adolescent. Psychotherapy.

Resumen: El objetivo de este ensayo es abordar la cuestión de cómo el sistema público puede garantizar el acceso al tratamiento psicoterapéutico de niños, niñas y adolescentes víctimas de violencia sexual, teniendo en cuenta que el desarrollo de psicopatologías está asociado, a corto y largo plazo, a la experiencia de violencia sexual. Se revisaron las principales leyes y directrices sobre la organización de los servicios públicos de asistencia social y de salud para víctimas de violencia sexual, así como los estudios sobre las derivaciones realizadas en dichos casos. Se constató que el servicio público de asistencia debe ofrecer atendimiento psicosocial, mientras que el servicio público de salud debe ofrecer psicoterapia. Sin embargo, los estudios sobre derivación de casos de violencia sexual contra niños, niñas y adolescentes indican bajos índices de derivación para servicios que ofrecen psicoterapia. Se discute dicha panorámica a partir de las directrices de atención al público realizado por el servicio público de salud, en el área de salud mental, así como a partir del número de estos servicios en Brasil. Se discuten alternativas con las que poder garantizar, en el sistema público de salud, el acceso al tratamiento psicoterapéutico de niños, niñas y adolescentes víctimas de violencia sexual.

Palabras-clave: Violencia sexual. Infancia. Adolescencia. Psicoterapia.

O objetivo deste ensaio é abordar a questão da garantia de acesso ao tratamento psicoterápico de crianças e adolescentes vítimas de violência sexual pelo sistema público. O desenvolvimento de psicopatologias em curto e em longo prazo associadas à experiência de violência sexual durante infância e adolescência indica a necessidade de se investir em políticas públicas de garantia de acesso à psicoterapia. Embora haja na legislação e nas diretrizes 
nacionais indicação de qual serviço deve fornecer atendimento psicoterápico às crianças e adolescentes vítimas de violência sexual, esse geralmente não ocorre devido a fatores apresentados no texto.

\section{Caracterização da violência sexual contra crianças e adolescentes}

A violência sexual contra crianças e adolescentes é definida pela World Health Organization (WHO) e pela International Society for Prevention of Child Abuse and Neglect (ISPCAN) como o envolvimento de uma criança ou adolescente em atividade sexual não compreendida totalmente, sendo esses incapazes de dar consentimento, ou para a qual não estão preparados devido a seu estágio desenvolvimental. Acrescenta-se o fato de que a violência sexual viola leis ou tabus da sociedade (WHO \& ISPCAN, 2006). No Brasil, a definição adotada pelo Ministério da Saúde (Brasil, 2002) é semelhante e há maior detalhamento das práticas consideradas violência sexual. Ela é compreendida, então, como todo e qualquer ato ou jogo sexual, seja ele em relação heterossexual seja em relação homossexual, no qual os perpetradores estão em estágio de desenvolvimento psicossexual mais adiantado do que a criança ou o adolescente. Por finalidade, define-se como prática que visa estimular sexualmente as vítimas ou utilizá-las para obtenção de satisfação sexual (dos perpetradores). Evidencia-se por meio de práticas eróticas e sexuais impostas às crianças ou aos adolescentes por violência física, ameaças ou indução de sua vontade. A violência sexual pode variar desde atos nos quais não há contato físico (e.g., voyeurismo, exibicionismo, produção de fotos), até diferentes tipos de ações que incluem contato físico com ou sem penetração. Inclui, ainda, situações de exploração sexual visando o lucro, tais como a exploração sexual e a pornografia (Brasil, 2002).

No final da década de 1990, a WHO reconheceu a violência sexual, bem como demais tipos de violência contra crianças e adolescentes, como um problema de saúde pública devido à sua prevalência, às suas consequências e aos gastos econômicos que acarretava. Em relação à violência sexual, sua prevalência entre crianças e adolescentes era, naquela década, de $7 \%$ a $34 \%$ entre meninas e de $3 \%$ a $29 \%$ entre meninos $(\mathrm{WHO}, 1999)$.

Dados epidemiológicos precisos acerca da violência sexual contra crianças e adolescentes são difíceis de serem obtidos devido à falta de sistematização das informações, bem como ao panorama de subnotificação desse tipo de violência. A falta de preparo dos profissionais e a falta de padronização das ferramentas de notificação podem ser citadas como fatores que favorecem essa situação. Além disso, os dados disponíveis são baseados, principalmente, em notificações, não abrangendo a totalidade de ocorrências de violência sexual, uma vez que existem casos que não são notificados.

Em nível mundial, a prevalência da violência sexual contra crianças e adolescentes em populações não clínicas (i.e., estudantes e população em geral) foi investigada por meio de um estudo de meta-análise (Pereda, Guilera, Forns \& Gómez-Benito, 2009). Foram analisados 65 artigos de 22 países diferentes, sendo a maioria $(n=12)$ proveniente dos Estados Unidos da América, além de três estudos canadenses, todos anteriores a 1997, e nenhum brasileiro. No total, 63 estudos analisados reportavam resultados sobre prevalência de violência sexual contra meninas e 37 contra meninos, sendo que um mesmo estudo poderia reportar resultados para ambos os sexos. O total da amostra analisada foi de 101.022 pessoas, sendo 37.094 do sexo masculino e 63.118 do sexo feminino. Após a análise dos resultados de todos os artigos, a média de prevalência de violência sexual foi de $7,9 \%$ para o sexo masculino e $19,7 \%$ para o sexo feminino, sendo essa diferença significativa $(p<0,05)$. $\mathrm{O}$ continente com maior prevalência foi o africano $(34,4 \%)$ e o com a menor, o europeu $(9,2 \%)$. Os continentes americano, asiático e oceânico alcançaram índices entre 10,1\% e $23,9 \%$. Tais resultados, conforme os autores, apresentam um panorama geral sobre a violência sexual mas devem ser utilizados com cautela devido a fatores como as diferentes definições de violência sexual e os diferentes métodos de coleta de dados empregados nos estudos analisados. 
No Brasil, o Programa Nacional de Enfrentamento da Violência Sexual contra Crianças e Adolescentes (Brasil, 2010), por meio do Disque Direitos Humanos (Disque 100), recebe notificações de casos de violência sexual contra crianças e adolescentes de todo o país. Periodicamente, tal programa divulga relatórios com informações sobre as notificações de violência sexual, sendo essa iniciativa uma das únicas sistematizações de dados epidemiológicos no Brasil. No entanto, esses relatórios incluem casos nos quais não há confirmação de violência sexual (i.e., suspeitas), sendo os resultados enviesados por esse motivo. Entre maio de 2003 e março de 2010, os dados divulgados evidenciam a predominância de casos de meninas vítimas de violência sexual em âmbito nacional. Dentre as 214.689 vítimas com sexo informado registradas nas notificações de violência sexual, negligência, violência física e psicológica, o percentual que mais diferiu entre as vítimas do sexo masculino e feminino foi no índice de violência sexual. Apenas a porcentagem foi divulgada, sendo $38 \%$ para o sexo masculino e $62 \%$ para o sexo feminino. Especificamente sobre os registros de violência sexual foi verificado que em todas as modalidades apresentadas (i.e., exploração sexual, tráfico de crianças e adolescentes, abuso sexual e pornografia), as vítimas do sexo feminino foram em maior número, obtendo o índice de $82 \%$ nas ocorrências de exploração sexual. Nas situações de violência sexual e pornografia, as vítimas do sexo masculino apresentaram aumento, mas ainda foram em menor porcentagem $(30 \%)$ do que as de sexo feminino $(70 \%)$.

Esses resultados indicam a ocorrência da violência sexual contra ambos os sexos, embora pareça haver mais casos notificados de vítimas do sexo feminino. Independentemente do sexo das vítimas, a ocorrência da violência sexual é um fator de risco para o desenvolvimento de sintomas e quadros psicopatológicos. É considerada, então, um estressor generalizado e as consequências apresentadas pelas vítimas dependerão da propensão delas para apresentá-las (Williams, 2002).

As consequências da violência sexual ocorrida na infância ou adolescência indicadas pela literatura são variadas, sendo as mais comuns, para ambos os sexos: ansiedade, raiva, dissociação, problemas interpessoais, além de psicopatologias como abuso de álcool e substâncias, depressão, transtornos alimentares, transtorno obsessivo-compulsivo, transtorno do estresse pós-traumático e transtorno de personalidade borderline (para uma revisão completa, consultar Hillberg, Hamilton-Giachritsis \& Dixon, 2011).

Em um estudo comparando vítimas de ambos os sexos não foram encontradas diferenças significativas quanto às consequências da violência sexual em longo prazo (Dube et al., 2005). Foi realizado um estudo retrospectivo de coorte com participação de 17.337 adultos americanos (54\% mulheres, $46 \%$ homens) vítimas de violência sexual na infância. Os participantes responderam a um questionário sobre disfunção familiar e violências durante a infância, além de questões relacionadas à saúde. Análises de regressão logística multivariadas foram utilizadas para investigar as possíveis relações entre violência sexual, saúde e problemas sociais (i.e., uso e abuso de drogas, doenças mentais e problemas atuais com o casamento e família), de acordo com o sexo da vítima. Os resultados indicaram que o impacto em longo prazo sobre as vítimas de violência sexual na infância foi semelhante para ambos os sexos. De acordo com os autores, esse resultado indica que meninos e meninas são vulneráveis às consequências da ocorrência de vitimizações na infância e que ambos necessitam de tratamento (Dube et al., 2005). No entanto, diferenças quanto a sintomas internalizantes e externalizantes, sendo os primeiros indicados como mais frequentes em meninas e os últimos, em meninos, são citadas pela literatura (Romano \& De Luca, 2001), evidenciando não haver consenso quanto a possíveis diferenças relacionadas às consequências da violência sexual para ambos os sexos.

As consequências da violência sexual, especialmente as de longo prazo, justificam o investimento em serviços de atendimento com o objetivo de aumentar a qualidade de vida de crianças e adolescentes vítimas e prevenir a ocorrência de psicopatologias na infância, adolescência e idade adulta. A ocorrência de sintomas e psicopatologias em longo prazo indica que a passagem de tempo após a violência sexual sem qualquer tipo 
de intervenção psicológica não é suficiente para reduzir tais sintomas. Por exemplo, em um estudo (Habigzang, Damásio \& Koller, 2013) com 49 meninas brasileiras entre 9 e 16 anos $(M=11.43)$ que participaram de uma grupoterapia cognitivo-comportamental para vítimas de violência sexual, os sintomas de depressão, ansiedade, estresse e transtorno do estresse pós-traumático foram investigados antes do início da intervenção. As meninas foram agrupadas conforme o tempo de espera para intervenção - (1) imediato, (2) um a seis meses e (3) mais de seis meses. Os resultados indicaram não haver diferenças significativas nos sintomas investigados entre as meninas dos três grupos formados conforme o tempo de espera para intervenção psicológica. Além disso, todas as meninas, independentemente do tempo de espera pela intervenção psicológica, apresentaram melhoras significativas nos sintomas de ansiedade, estresse e transtorno do estresse pós-traumático, além de estabilização dos sintomas de depressão quando comparados os escores pré e pós-intervenção (Habigzang et al., 2013).

\section{Políticas nacionais de proteção e de atendimento a crianças e adolescentes vítimas de violência sexual}

No Brasil, as políticas de proteção e atendimento a crianças e adolescentes foram sedimentadas pela lei número 8.069, de 13 de julho de 1990, conhecida como Estatuto da Criança e do Adolescente - ECA (Brasil, 1990a). Em seu quinto artigo, o ECA determina que "nenhuma criança ou adolescente será objeto de qualquer forma de negligência, discriminação, exploração, violência, crueldade e opressão, punido na forma da lei qualquer atentado, por ação ou omissão, aos seus direitos fundamentais", sendo "dever de todos prevenir a ocorrência de ameaça ou violação dos direitos da criança e do adolescente" (artigo 70). Quando há violação desses direitos é necessário comunicar a autoridade competente para que as medidas de proteção sejam adotadas.

Em relação aos atendimentos, o ECA (Brasil, 1990a) estabelece que "a política de atendimento dos direitos da criança e do adolescente far-se-á através de um conjunto articulado de ações governamentais e não governamentais da união, dos estados, do Distrito Federal e dos municípios" (artigo 86). Especificamente acerca de situações de violência sexual, a linha de ação da política de atendimento consiste em "serviços especiais de prevenção e atendimento médico e psicossocial às vítimas de negligência, maus-tratos, exploração, abuso, crueldade e opressão" (artigo 87). Além disso, o Plano Nacional de Enfrentamento da Violência Sexual, lançado em 2000, e o Programa de Ações Integradas e Referenciais de Enfrentamento à Violência Sexual contra Crianças e Adolescentes no Território Brasileiro (PAIR), implementado em 2003, estão estruturados em seis eixos estratégicos (i.e., análise da situação, mobilização e articulação, defesa e responsabilização, atendimento, prevenção e protagonismo infanto-juvenil), sendo um específico para o atendimento. $\mathrm{O}$ eixo atendimento visa garantir o atendimento especializado e em rede às crianças e aos adolescentes vítimas de violência sexual e respectivas famílias (Bispo, Luz, Gadelha \& Paiva, 2011).

Os serviços de atendimento às situações de violência sexual no Brasil estão inclusos no Sistema Único de Assistência Social (SUAS). Esse é organizado pela Política Nacional de Assistência Social - PNAS (Brasil, 2004a), a qual é baseada na Lei Orgânica da Assistência Social - LOAS (Brasil, 1993) e coordenada pelo Ministério do Bem-Estar Social. Essa lei estabelece normas e critérios para organização da assistência social.

Na organização dos serviços de assistência social devem ser criados programas de amparo às pessoas que vivem em situação de rua e às crianças e adolescentes em situação de risco pessoal e social, em cumprimento a Constituição Federal e ao ECA (Artigo 23) (Brasil, 1993). Além desses, o PNAS (Brasil, 2004a) indica que constituem usuários dos serviços sociais cidadãos e grupos que se encontram em situações de vulnerabilidade e risco, sendo os serviços sociais divididos conforme a complexidade das situações e população que atendem: proteção social básica, proteção social especial, dividida em média complexidade e alta complexidade (Brasil, 2004a). 
A proteção social básica visa prevenir situações de risco por meio do desenvolvimento de potencialidades e aquisições, bem como o fortalecimento de vínculos familiares e comunitários, tendo como principal representante, mas não único, os Centros de Referência em Assistência Social (CRAS). É destinada à população em situação de vulnerabilidade social decorrente de pobreza, privação (i.e., ausência de renda, precário ou nulo acesso aos serviços públicos, dentre outros) e/ou fragilização de vínculos afetivos - relacionais e de pertencimento social (i.e., discriminações etárias, étnicas, de gênero ou por deficiências, dentre outras). Sua atuação compreende o desenvolvimento de serviços, programas e projetos locais de acolhimento, convivência e socialização de famílias e de indivíduos, incluindo pessoas com deficiência. Seu objetivo é superar as condições de vulnerabilidade e prevenir situações que indicam risco potencial (Brasil, 2004a).

A proteção social especial é a modalidade de atendimento assistencial destinada a famílias e indivíduos que se encontram em situação de risco pessoal e social. É dividida em média e alta complexidade. É distinta da proteção social básica por se tratar de um atendimento dirigido às situações de violação de direitos, tal como a violência sexual. Isso indica a necessidade de desencadear estratégias de atenção sociofamiliar que visem à reestruturação do grupo familiar e à elaboração de novas referências morais e afetivas. Destina-se a famílias e indivíduos que se encontram em situação de risco pessoal e social por ocorrência de abandono, maus tratos físicos e/ou psíquicos, violência sexual, uso de substâncias psicoativas, cumprimento de medidas socioeducativas, situação de rua, situação de trabalho infantil, dentre outras. Os serviços da proteção social especial devem abranger desde o provimento de acesso a serviços de apoio e sobrevivência até sua inclusão em redes sociais de atendimento e de solidariedade (Brasil, 2004a).

Os serviços de proteção social especial de média complexidade são aqueles que oferecem atendimento às famílias e indivíduos com direitos violados mas cujos vínculos familiares e comunitários não foram rompidos. Sendo assim, requerem maior estruturação técnico-operacional e atenção especializada e mais individualizada e/ou acompanhamento sistemático e monitorado por meio de serviços de orientação e apoio sociofamiliar, plantão social, abordagem de rua, dentre outros. Têm como principal serviço o Centro de Referência em Assistência Social (CREAS), visando a orientação e o convívio sociofamiliar e comunitário (Brasil, 2004a).

Por fim, os serviços de proteção social especial de alta complexidade são aqueles que garantem a proteção integral (i.e., moradia, alimentação, higienização e trabalho protegido) para famílias e indivíduos que se encontram sem referência e/ou em situação de ameaça, necessitando ser retirados de seu núcleo familiar e/ou comunitário. Os serviços de proteção social especial de alta complexidade mais comuns são os de atendimento integral institucional, casa lar, república, casa de passagem, albergue, família substituta, família acolhedora, medidas socioeducativas restritivas e privativas de liberdade (i.e., semiliberdade, internação provisória e sentenciada) e trabalho protegido (Brasil, 2004a).

Sendo assim, crianças e adolescentes vítimas de violência sexual devem ser atendidas em serviços de proteção social especial tendo em vista a ocorrência de violação de direitos. Caso os agressores sejam afastados, as vítimas recebem atendimento via proteção social especial de média complexidade, principalmente nos CREAS. Caso o acolhimento institucional da vítima seja necessário, ela passará a ser atendida em serviços de proteção social especial de alta complexidade. Os atendimentos em serviços de proteção social especial de média e alta complexidade não são excludentes, pois muitas vítimas em acolhimento institucional frequentam serviços como o CREAS.

\section{Psicoterapia nos serviços públicos de atendimento a crianças e adolescentes vítimas de violência sexual}

A análise das referências para atuação de psicólogos no serviço de proteção social a crianças e adolescentes vítimas de violência sexual publicadas pelo Conselho Federal de Psicologia (CFP, 2009) indica que esse profissional deve oferecer atendimento 
psicossocial às vítimas e suas famílias estando, assim, em consonância com as diretrizes do SUAS. O atendimento psicossocial, segundo esse documento, difere da psicoterapia pela forma de intervenção e pelos seus objetivos. Consiste, então, em um

conjunto de procedimentos técnicos especializados, com o objetivo de estruturar ações de atendimento e de proteção a crianças e adolescentes, proporcionando-lhes condições para o fortalecimento da autoestima, o restabelecimento de seu direito à convivência familiar e comunitária em condições dignas de vida e possibilitando a superação da situação de violação de direitos, além da reparação da violência sofrida (CFP, 2009, p. 39).

Ainda segundo o documento do CFP (2009, p. 50), os serviços de psicoterapia "têm o seu lugar na atenção à saúde, mais especificamente nos serviços de saúde mental", sendo o(a) psicólogo(a) do CREAS responsável por avaliar a necessidade de encaminhamento para esses serviços. Segundo artigo 10 da resolução 10/2000 do CFP (2000), a psicoterapia é

\footnotetext{
processo científico de compreensão, análise e intervenção que se realiza através da aplicação sistematizada e controlada de métodos e técnicas psicológicas reconhecidos pela ciência, pela prática e pela ética profissional, promovendo a saúde mental e propiciando condições para o enfrentamento de conflitos e/ou transtornos psíquicos de indivíduos ou grupos.
}

Sendo assim, vítimas de violência sexual que apresentam quadros psicopatológicos deveriam receber, além de acompanhamento psicossocial, tratamento psicoterápico sempre que necessário. Dessa forma o direito de portadores de transtornos mentais de receber o "melhor tratamento do sistema de saúde, consentâneo às suas necessidades" (Brasil, 2001, parágrafo único, item I) é respeitado. Os tratamentos psicossociais e psicoterápicos não são excludentes, pois, apesar de possuírem alguns objetivos diferentes, compartilham o objetivo final de propiciar maior qualidade de vida para os usuários.

No Brasil, os serviços de saúde mental estão inclusos no Sistema Único de Saúde (SUS), representados pelos Centros de Atenção
Psicossocial (CAPS). O SUS foi criado por meio da Constituição Federal (Brasil, 1988) e regulamentado pela lei 8.080 , de 1990, baseado na premissa de que o provimento do acesso à saúde é dever do Estado, sendo a saúde compreendida como o bem-estar físico, mental e social (artigo 2) (Brasil, 1990b). É constituído por um conjunto de ações e serviços de saúde prestados por órgãos e instituições públicas federais, estaduais e municipais de forma gratuita aos cidadãos (artigo 4) (Brasil, 1990b). Em relação à saúde mental, no ano de 1992 foram criados os CAPS (Brasil, 1992).

O CAPS, também chamado de Núcleo de Atenção Psicossocial, é um serviço de saúde para tratamento de pessoas que sofrem com transtornos mentais, psicoses, neuroses graves e demais quadros. Seus usuários são, preferencialmente, "pessoas com transtornos mentais severos e/ou persistentes, ou seja, pessoas com grave comprometimento psíquico". O atendimento no CAPS ocorre por meio de procura direta do usuário ou por seu encaminhamento por qualquer serviço de saúde (Brasil, 2004b).

Embora sejam divididos conforme o número de habitantes nos municípios onde se localizam (i.e., devendo sempre ser maior do que 200 mil), horários de funcionamento e usuários a quem se destinam (i.e., adultos, crianças e adolescentes ou usuários de álcool e outras drogas), todos os CAPS (i.e., CAPS I, II e III, CAPSi e CAPSad) devem oferecer os seguintes atendimentos: individual (i.e., prescrição de medicamentos, psicoterapia e orientação); grupo (i.e., oficinas terapêuticas, expressivas, geradoras de renda, de alfabetização, culturais; grupos terapêuticos, atividades esportivas e de suporte social; grupos de leitura e debate, bem como de confecção de jornal); familiar (i.e., atendimento nuclear e a grupo de familiares; atendimento individualizado a familiares; visitas domiciliares; atividades de ensino e de lazer com familiares). Tais atendimentos são divididos conforme sua intensidade: intensivo (i.e., atendimento diário, oferecido quando a pessoa se encontra com grave sofrimento psíquico, em situação de crise ou dificuldades intensas no convívio social e familiar, precisando de atenção contínua, podendo ser domiciliar, se necessário); semi-intensivo (i.e., quando o sofrimento e a desestruturação 
psíquica da pessoa diminuíram, melhorando as possibilidades de relacionamento, mas a pessoa ainda necessita de atenção direta da equipe para se estruturar e recuperar sua autonomia, podendo ser domiciliar, se necessário, porém, limitado até 12 dias no mês); e não intensivo (i.e., oferecido quando a pessoa não precisa de suporte contínuo da equipe para viver em seu território e realizar suas atividades na família e/ou no trabalho, podendo ser atendida até três dias no mês e à domicílio - Brasil, 2004b).

Recentemente, em agosto de 2013, a Presidência da República sancionou a lei $n$. 12.845, a qual dispõe sobre o atendimento imediato e integral - médico, psicológico e social - às pessoas em situação de violência sexual em hospitais gerais. Em seu primeiro artigo, essa lei indica que

os hospitais devem oferecer às vítimas de violência sexual atendimento emergencial, integral e multidisciplinar, visando ao controle e ao tratamento dos agravos físicos e psíquicos decorrentes de violência sexual, e encaminhamento, se for o caso, aos serviços de assistência social (Brasil, 2013).

A análise das diretrizes para os serviços de atendimento a crianças e adolescentes vítimas de violência sexual indica, portanto, que essas devem receber atendimento psicossocial no CREAS (SUAS) e, quando o(a) psicólogo(a) desse serviço julgar necessário, deve-se proceder encaminhamento para um serviço de saúde mental (SUS). Dessa forma, a avaliação diagnóstica cuidadosa, verificando sintomas típicos em crianças e adolescentes vítimas de violência sexual, deveria ser sistematizada e protocolada como prática dos psicólogos nos CREAS para subsidiar o encaminhamento para psicoterapia no sistema de saúde sempre que necessário. Sintomas de transtornos de ansiedade, de humor, alimentares e disruptivos, comuns em crianças e adolescentes vítimas de violência sexual, devem ser avaliados de forma objetiva por meio de instrumentos que visem investigá-los (Hohendorff \& Habigzang, 2014). A entrevista clínica estruturada com base no Manual Diagnóstico e Estatístico de Transtornos Mentais (DSM-IV) para transtornos na infância e adolescência (K-SADS-PL, Brasil, 2003; Kaufmanet al., 1997) é um instrumento útil para tal avaliação.
O CAPSi (Centro de Atenção Psicossocial Infantil) é o serviço do SUS de referência para atendimento em saúde mental, sendo que sua diretriz indica que deve receber, preferencialmente, casos de transtornos mentais severos e/ou persistentes. Tendo em vista que nos documentos que fundamentam a ação dos CAPS não são apresentados critérios para a consideração de transtornos mentais severos e/ou persistentes, tal julgamento acaba sendo feito pelo profissional responsável pelo atendimento. Casos de crianças e adolescentes vítimas de violência sexual que apresentam sintomas psicopatológicos mas que não são considerados severos e/ou persistentes não possuem nenhum serviço de referência de saúde mental para seu atendimento psicoterápico. Assim, verifica-se um descompasso entre o conhecimento científico existente sobre as consequências da violência sexual, que indica o desenvolvimento em curto e longo prazo de psicopatologias e a necessidade de psicoterapia como principal recurso para redução de sintomas, e as políticas públicas nacionais, que não determinam claramente o acesso dessa população clínica à psicoterapia.

Os estudos sobre os encaminhamentos adotados em casos de violência sexual contra crianças e adolescentes indicam baixa frequência de encaminhamentos a serviços de saúde mental (Habigzang, Azevedo, Koller \& Machado, 2006; Habigzang, Ramos \& Koller, 2011; Pelisoli, Pires, Almeida \& Dell'Aglio, 2010). Quando esse encaminhamento ocorre, não há informação precisa sobre qual o serviço responsável, sendo citados apenas como tratamento psicológico, serviço multidisciplinar para atendimento psicológico (Habigzang et al., 2006) e avaliação psicológica (Habigzang et al., 2011).

O baixo número de encaminhamentos de vítimas de violência sexual aos serviços de saúde mental pode ser explicado, então: (a) pela restrição quanto à severidade e persistência dos transtornos mentais para atendimento nos CAPS (Brasil, 2004b); (b) pela falta de capacitação profissional para intervenção em casos de violência sexual, a qual foi evidenciada pelo CFP (2009), possivelmente devido à abordagem escassa desse assunto em cursos de graduação (Gonçalves \& Ferreira, 2002), o que pode fazer com que profissionais não aceitem encaminhamentos; (c) pelo baixo 
número de CAPSi existentes no país - em 2011 havia apenas 149 (Brasil, 2012).

Recentemente, a Fundação Oswaldo Cruz - FIOCRUZ (Deslandes, 2013) realizou uma pesquisa sobre as estratégias municipais para o enfrentamento da violência sexual contra crianças e adolescentes em quatro capitais brasileiras (i.e., Belém, PA, Campo Grande, MS, Fortaleza, CE e Porto Alegre, RS), sendo que somente os resultados de Porto Alegre foram divulgados até o momento. Os resultados indicaram ser escasso o número de serviços de saúde mental para vítimas de violência sexual no município (apenas dois), sendo recomendado, então, investimento nesse tipo de serviço. Possivelmente, esse resultado e recomendação se repetirão nas demais capitais, dado o baixo número de serviços públicos de saúde mental em nosso país (Brasil, 2012).

Diante do panorama de falta de um serviço público nacional que ofereça psicoterapia para vítimas de violência, essa tende a ser realizada por serviços de saúde mental geralmente criados pela administração estadual ou municipal, muitas vezes em parceria com instituições de ensino superior, para absorver a demanda de crianças e adolescentes que necessitam. Sendo assim, não há conhecimento amplo de quais são os serviços públicos que oferecem atendimento em saúde mental para crianças e adolescentes vítimas de violência sexual no território brasileiro, o que dificulta o planejamento de intervenções e a execução de encaminhamentos.

Em 2006, a WHO e a ISPCAN publicaram um guia no qual indicaram as quatro principais diretrizes para as intervenções psicoterápicas para crianças e adolescentes vítimas de violência, dentre elas a sexual. As quatro diretrizes são: (a) as intervenções devem ser baseadas em evidências, ou seja, ao planejar uma intervenção psicoterápica devem-se buscar subsídios em resultados de pesquisas e em manuais de psicoterapia para saber o que é adequado (ou não) incluir no tratamento; (b) as intervenções devem ser orientadas por objetivos, ou seja, juntamente com os pacientes deve-se estabelecer aonde se quer chegar com a psicoterapia; (c) as intervenções devem ter abordagem estruturada, ou seja, com início, meio e fim delimitados a priori, sabendo-se o que será abordado em cada etapa e por que será abordado; e (d) as intervenções devem abordar estratégias para manejo das consequências emocionais e comportamentais decorrentes da violência, ou seja, técnicas específicas para diminuição de sintomas comuns em vítimas de violência e que tenham, conforme o item a, evidências de adequação e efetividade.

Os psicólogos que atuam na rede de atendimento a crianças e adolescentes vítimas de violência sexual devem estar capacitados para oferecer psicoterapia de acordo com as diretrizes da WHO e da ISPCAN. No entanto, a falta de capacitação desses profissionais é uma realidade indicada pelo próprio CFP (2009), resultado da abordagem escassa da violência sexual em cursos de graduação (Gonçalves \& Ferreira, 2002), incluindo o de Psicologia. Dessa forma, torna-se necessário o investimento em tecnologias de capacitação profissional para esses profissionais, visando capacitá-los para a realização de avaliações psicológicas, bem como tratamentos psicoterápicos baseados em evidências para crianças e adolescentes vítimas de violência sexual (Freitas \& Habigzang, 2014).

No cenário internacional há vários estudos sobre tratamento psicoterápico para crianças e adolescentes vítimas de violência sexual (e.g., Cohen, Deblinger, Mannarino \& Steer, 2004; Cohen, Mannarino \& Knudsen, 2005; Hetzel-Riggin, Brausch \& Montgomery, 2007). A análise desses estudos permite que se verifiquem tratamentos psicoterápicos mais efetivos e eficazes para atendimento de crianças e adolescentes vítimas de violência sexual.

Abordagens psicoterápicas cognitivo-comportamentais focadas no trauma e centradas na criança foram avaliadas em um estudo com 229 crianças e adolescentes entre 8 e 14 anos vítimas de violência sexual (Cohen et al., 2004). Os participantes foram divididos randomicamente entre as duas abordagens psicoterápicas. Ambas as abordagens tiveram duração de 12 sessões semanais no formato individual. Instrumentos para avaliação sintomatológica foram utilizados antes e após a intervenção. Ao se compararem os grupos, aqueles que receberam terapia cognitivo-comportamental focada no trauma apresentaram melhoras 
significativas em relação ao transtorno do estresse pós-traumático, depressão, atribuições de responsabilidade pela violência sexual, vergonha e problemas de comportamento.

Em outro estudo, a manutenção dos resultados de duas abordagens psicoterápicas foi avaliada com a participação de 82 crianças e adolescentes vítimas de violência sexual com idade entre 8 e 14 anos (Cohen et al., 2005). Os participantes foram divididos randomicamente em dois grupos distintos conforme a abordagem terapêutica utilizada: terapia cognitivo-comportamental focada no trauma e terapia de suporte não diretiva, ambas aplicadas no formato individual e com duração de 12 sessões semanais. Instrumentos psicológicos foram utilizados para avaliação de sintomas no período pré-intervenção, pós-intervenção, 6 e 12 meses após o término da intervenção. A comparação entre as abordagens utilizadas indicou que a terapia cognitivo-comportamental focada no trauma apresentou resultados superiores na produção e manutenção de melhoras terapêuticas em sintomas de ansiedade, depressivos, problemas sexuais e dissociação 6 meses após a intervenção e no transtorno do estresse pós-traumático e dissociação 12 meses após a intervenção (Cohen et al., 2005).

Em suma, esses dois estudos indicam a abordagem cognitivo-comportamental como tratamento de escolha, pois é a mais eficaz na redução de sintomas e seus resultados se mantêm após o encerramento do tratamento. Além deles, um estudo de meta-análise também indicou a eficácia da abordagem cognitivo-comportamental (Hetzel-Riggin et al., 2007). Diferentes bancos de dados foram consultados com o objetivo de selecionar estudos sobre os efeitos de diferentes tratamentos em problemas apresentados por crianças e adolescentes vítimas de violência sexual. Um total de 28 estudos de língua inglesa publicados entre 1975 e 2004 foi selecionado. Os resultados indicaram a efetividade de qualquer forma de tratamento em comparação a não receber tratamento algum. Houve heterogeneidade quanto à eficácia de diferentes abordagens analisadas em relação aos problemas apresentados pelas vítimas. No entanto, a abordagem cognitivo-comportamental, tanto no formato individual quanto no grupal, obteve eficácia em maior número de problemas, sendo eles estresse psicológico, problemas comportamentais e baixo autoconceito (Hetzel-Riggin et al., 2007).

No Brasil também há estudos sobre intervenções psicoterápicas para crianças e adolescentes vítimas de violência sexual (Habigzang et al., 2009; Hohendorff, Salvador-Silva, Andrade, Habigzang \& Koller, 2014; Lucânia, Valério, Barison \& Miyazaki, 2009). Os resultados desses estudos são semelhantes aos dos estudos internacionais, indicando a terapia cognitivo-comportamental como o tratamento de escolha.

A abordagem cognitivo-comportamental foi utilizada para o tratamento de uma menina de 13 anos vítima de violência sexual (Lucânia et al., 2009). A intervenção foi composta por 45 sessões. As cinco primeiras sessões tiveram como objetivo o estabelecimento de vínculo e o levantamento de informações sobre o caso. Uma entrevista semidirigida, os critérios diagnósticos para transtorno do estresse pós-traumático e um instrumento psicológico para avaliação de sintomatologia depressiva foram utilizados. A partir da sexta sessão até a 35ª , os sintomas foram trabalhados e técnicas cognitivas e comportamentais foram utilizadas. A avaliação final foi realizada nas três sessões seguintes e o follow-up foi conduzido nas cinco últimas sessões. Por meio da avaliação do processo de psicoterapia, do relato da menina e de seus comportamentos foi concluído que a intervenção cognitivo-comportamental resultou em melhora nos sintomas do transtorno do estresse pós-traumático, depressão, dificuldades de comportamento e escolares.

Além do estudo de Lucânia et al. (2009), há estudos brasileiros sobre um modelo de grupoterapia cognitivo-comportamental que também fornecem evidências de efetividade da abordagem cognitivo-comportamental para tratamento de meninas vítimas de violência sexual (Habigzang et al., 2009). O modelo é composto por 16 sessões, divididas em três etapas (i.e., psicoeducação - seis sessões; treino de inoculação do estresse - quatro sessões; prevenção à recaída - seis sessões). A avaliação desse modelo foi realizada por meio de um ensaio clínico com delineamento pré e pós, com a participação de 40 meninas entre 9 e 16 anos que haviam passado por, 
no mínimo, um episódio de violência sexual intra ou extrafamiliar (Habigzang et al., 2009). As participantes foram avaliadas em quatro tempos distintos do processo de grupoterapia: pré-teste (i.e., avaliação inicial), pós-teste 1 (i.e., após psicoeducação), pós-teste 2 (i.e., após treino de inoculação do estresse) e pós-teste 3 (i.e., após prevenção à recaída) em relação a sintomas de depressão, ansiedade, transtorno do estresse pós-traumático, estresse e crenças distorcidas sobre a ocorrência da violência sexual. Os resultados do estudo indicaram a efetividade do modelo de grupoterapia cognitivo-comportamental para meninas vítimas de violência sexual na redução de sintomas de depressão, ansiedade, estresse e transtorno do estresse pós-traumático, além de contribuir para a redução de crenças distorcidas relacionadas à culpa pela violência sexual, confiança nas outras pessoas e credibilidade (Habigzang et al., 2009).

Posteriormente foi verificado o impacto do modelo de grupoterapia cognitivo-comportamental para meninas vítimas de violência sexual aos 6 e 12 meses após o seu término. Nesses períodos, 35 meninas foram avaliadas. Os resultados indicaram redução de sintomas de ansiedade, estresse e transtorno do estresse pós-traumático, sendo essa redução mantida aos 6 e 12 meses após o seu término (Habigzang et al., 2013).

Diante dos resultados da avaliação do modelo de grupoterapia cognitivo-comportamental para tratamento de meninas vítimas de violência sexual, esse foi adaptado para utilização com meninos. Nessa adaptação, o formato da intervenção passou a ser individual devido ao número reduzido de encaminhamentos de casos de meninos vítimas de violência sexual para psicoterapia. A aplicação do modelo adaptado foi avaliada por meio de um estudo qualitativo com a participação de três meninos com idades entre 8 e 16 anos vítimas de violência sexual intra e extrafamiliar. Esses meninos foram avaliados antes e após a intervenção quanto a sintomas de depressão, estresse, ansiedade, transtornos de déficit de atenção e hiperatividade, desafiador opositivo, de conduta e de estresse pós-traumático, dependência/abuso de álcool e outras substâncias, bem como acerca de crenças distorcidas sobre a ocorrência da violência sexual. Os resultados do estudo indicaram adesão dos meninos à intervenção, diminuição nos sintomas de depressão e estresse para os três participantes, sendo que nos demais sintomas (i.e., ansiedade, transtorno de déficit de atenção e hiperatividade, transtorno de conduta e desafiador-opositivo e transtorno do estresse pós-traumático) houve variabilidade entre os participantes. Além disso, foi constatada a formação de uma boa aliança terapêutica entre os psicoterapeutas e os meninos, facilitando que esses se autorrevelassem (Hohendorff et al., 2014).

Estudos nacionais e internacionais sobre abordagens psicoterápicas para tratamento de crianças e adolescentes indicam, portanto, a abordagem cognitivo-comportamental como aquela que possui mais evidências sobre sua efetividade e eficácia. Os tratamentos avaliados nesses estudos geralmente são manualizados, ou seja, possuem um roteiro predefinido quanto à estrutura das sessões, técnicas utilizadas e objetivos a serem alcançados. Os tratamentos manualizados permitem maior controle do processo psicoterápico bem como auxiliam psicoterapeutas e pacientes a avaliarem constantemente o seu progresso. Embora sejam manualizados, esses tratamentos não são rígidos e podem ser adaptados às necessidades dos pacientes sempre que necessário.

A capacitação de psicólogos para aplicação de tratamentos manualizados tende a ser facilitada devido às suas características, ou seja, definição da estrutura das sessões, técnicas utilizadas e objetivos a serem alcançados. Dessa forma, as tecnologias de capacitação profissional devem incluir os resultados de estudos sobre intervenções psicoterápicas para crianças e adolescentes vítimas de violência sexual realizados no Brasil, pois esses indicam quais estratégias são mais efetivas. Devem ser ministradas por experts no assunto, com reconhecida experiência na pesquisa e prática clínica para essa população. O conteúdo deve incluir aspectos teóricos da violência sexual (e.g, dados epidemiológicos, dinâmica, consequências), bem como aspectos da prática para avaliação psicológica e intervenção clínica por meio do treinamento de técnicas utilizadas com crianças e adolescentes vítimas de violência sexual. Além disso, devem ser estabelecidos planos de implementação, acompanhamento e supervisão das intervenções psicoterápicas 
realizadas pelos profissionais capacitados (Freitas \& Habigzang, 2014).

A psicoterapia é um mediador do impacto da violência sexual sofrida na infância e adolescência. Vítimas que receberam intervenções adequadas após a revelação da violência sexual apresentaram redução de sintomas de depressão, ansiedade, transtorno do estresse pós-traumático e alteraram crenças e comportamentos disfuncionais decorrentes da violência (Cohen et al., 2004, 2005; Habigzang et al., 2013, 2009; Hetzel-Riggin et al., 2007). Sabe-se que a presença de tais sintomas na infância e adolescência pode prejudicar o desenvolvimento cognitivo, afetivo, social e acadêmico. As consequências negativas podem perdurar ao longo de toda a vida dos indivíduos. Dessa forma, a garantia de acesso de crianças e adolescentes vítimas de violência sexual a intervenções psicoterápicas com evidências de efetividade tem o potencial de reduzir custos com o tratamento de efeitos de longo prazo. Além disso, o tratamento psicoterápico ainda na infância pode reduzir riscos de revitimizações e desenvolvimento de comportamentos agressivos que contribuem para manutenção de ciclos de violência no contexto familiar.

\section{Conclusão}

A baixa frequência de encaminhamentos a serviços de saúde mental é incompatível com o conhecimento atual sobre as repercussões psicopatológicas da violência sexual para crianças e adolescentes vítimas. Tal panorama pode ser explicado parcialmente pela restrição quanto à severidade e persistência dos transtornos mentais de crianças e adolescentes para atendimento no CAPS (Brasil, 2004b), bem como pela falta de capacitação profissional para avaliação e intervenção em casos de violência sexual (CFP, 2009) e, por fim, pelo baixo número de CAPSi existentes no país - em 2011 havia apenas 149 (Brasil, 2012). Dessa forma, identifica-se uma lacuna referente ao atendimento contínuo de saúde mental para crianças e adolescentes vítimas de violência sexual que apresentam psicopatologias, embora esse seja um direito previsto em lei em nosso país (Brasil, 2001).
A responsabilidade pela política de atendimento dos direitos da criança e do adolescente, segundo o ECA (Brasil, 1990a), é de competência governamental, não governamental, da união, dos estados, do Distrito Federal e dos municípios, cabendo a esses a articulação necessária. Embora importante, a nomeação de diferentes instâncias responsáveis pelas políticas de atendimento pode acabar diluindo tal responsabilidade a ponto de nenhuma delas se perceber realmente responsável.

A questão, então, não é especificamente rejeitar o que preconiza o ECA (Brasil, 1990a) em relação à política de atendimento dos direitos da criança e do adolescente ser feita por meio de um conjunto articulado de ações governamentais e não governamentais, da União, dos estados, do Distrito Federal e dos municípios mas sim buscar nomear uma instância responsável por essa articulação. A articulação necessária à política de atendimento dos direitos da criança e do adolescente poderia, então, ter como instância responsável a união, a qual, além de aumentar o número de CAPSi no país, disponibilizaria tecnologias de capacitação profissional para psicólogos da rede de atendimento a crianças e adolescentes vítimas de violência sexual. É necessário, ainda, delimitar claramente o escopo das intervenções psicossociais e psicoterápicas, pois as definições existentes não são suficientemente claras quanto aos objetivos, método e técnicas de cada uma. Dessa forma, psicólogos do SUAS e SUS saberão qual seu papel diante de casos de violência sexual contra crianças e adolescentes, podendo intervir de forma adequada.

Intervenções para fortalecer o papel protetivo da família, para melhorar as relações familiares, reduzir o sentimento de estigmatização e culpa das vítimas e promover a reinserção social das crianças e adolescentes poderiam ser atribuídas ao atendimento psicossocial. Por outro lado, a reestruturação da memória traumática, redução de sintomas psicopatológicos, resolução de problemas decorrentes da violência sexual, melhora da autoestima e aprendizagem de comportamentos protetivos poderiam ser objetivos do atendimento psicoterápico. Dessa forma, psicólogos de SUAS e SUS teriam intervenções complementares que efetivamente contribuíssem para redução do 
impacto da violência sexual. É necessário que se utilizem práticas baseadas em evidências e que os sistemas públicos de atendimento desenvolvam estratégias para avaliar a efetividade de suas intervenções. Nessa direção verifica-se a necessidade de maior articulação entre pesquisa e prática em Psicologia.

Outra estratégia para modificar o panorama atual é a mudança nos currículos de graduação em Psicologia, objetivando a inclusão da temática da violência sexual contra crianças e adolescentes em disciplinas específicas ou já existentes. Sabe-se que a abordagem da violência sexual nos cursos de graduação é escassa (Gonçalves \& Ferreira, 2002), o que acarreta falta de preparo e interesse profissional para o trabalho com essa temática.

Ao incluir a temática da violência sexual contra crianças e adolescentes nos cursos de graduação em Psicologia, psicólogos(as) com maior conhecimento e mais bem preparados(as) para o trabalho com vítimas serão formados. Esses profissionais, ao participarem de tecnologias sociais de capacitação sobre violência sexual contra crianças e adolescentes, terão uma base de conhecimentos para atuação em órgãos de proteção, seguindo intervenções psicoterápicas adequadas e alinhadas às diretrizes da WHO e da ISPCAN (WHO, 2006). 


\section{Jean Von Hohendorff}

'Doutorando em Psicologia pela Universidade Federal do Rio Grande do Sul, Porto Alegre, RS. Brasil. E-mail: jhohendorff@gmail.com

\section{Luísa Fernanda Habigzang}

${ }^{2}$ Doutora em Psicologia pela Universidade Federal do Rio Grande do Sul, Porto Alegre Brasil. Docente da Pontifícia Universidade Católica do Rio Grande do Sul, Porto Alegre, RS. Brasil. E-mail: habigzang.luisa@gmail.com

\section{Silvia Helena Koller}

${ }^{3}$ Doutora em Educação pela Pontifícia Universidade Católica do Rio Grande do Sul.

Docente da Universidade Federal do Rio Grande do Sul, Porto Alegre, RS. Brasil. E-mail:

silvia.koller@pq.cnpq.br

\section{Endereço para envio de correspondência:}

Universidade Federal do Rio Grande do Sul (UFRGS), Instituto de Psicologia. Rua Ramiro Barcelos, 2600, sala 104. CEP 90035-003 - Porto Alegre, RS - Brasil.

Recebido 18/02/2014, 1a Reformulação 24/09/2014, 2ª Reformulação 21/10/2014, Aprovado 05/12/2014. 
Bispo, E., Luz, F., Gadelha, G., \& Paiva, L. (2011). Metodologia do PAIR. In Secretária de Direitos Humanos. Material Didático - Programa de Ações Integradas e Referenciais de Enfrentamento da Violência Sexual Infantojuvenil no Território Brasileiro - Pair - Conteúdos para capacitação (pp. 105-123). Recuperado em 09 de setembro de 2013, de http://pair.ledes.net/gestor/titan. php?target $=$ openFile\&fileld $=1108$

Brasil. (1988). Constituição da República Federativa do Brasil de 1988. Recuperado em 09 de setembro de 2013, de http://www.planalto. gov.br/ccivil_03/constituicao/constituicao.htm

Brasil. (1990a). Lei no 8.069, de 13 de julho de 1990. Diário Oficial da União, Brasília, DF. Recuperado em 09 de setembro de 2013, de http://www.planalto.gov.br/ccivil_03/leis/ L8069.htm

Brasil. (1990b). Lei no 8.080, de 19 de setembro de 1990. Diário Oficial da União, Brasília, DF. Recuperado em 09 de setembro de 2013, de http://www.planalto.gov.br/ccivil_03/leis/ I8080.htm

Brasil. (1992). Portaria $n^{\circ} 224 / M S$, de 29 de janeiro de 1992. Diário Oficial da União, Brasília, DF. Recuperado em 09 de setembro de 2013: http://www.mp.sc.gov.br/portal/site/ conteudo/cao/ccf/quadro\%20sinotico\%20sus/ portaria\%20sas-ms\%20n\%C2\%BA\%2022492\%20-\%20diretrizes\%20e\%20normas $\% 20$ saude\%20mental.pdf

Brasil. (1993). Lei no 8.742, de 7 de dezembro de 1993. Diário Oficial da União, Brasília, DF. Recuperado em 09 de setembro de 2013, de http://www.planalto.gov.br/ccivil_03/leis/ I8742.htm

Brasil. (2001). Lei no 10.216, de 6 de abril de 2001. Diário Oficial da União, Brasília, DF. Recuperado em 09 de dezembro de 2013, de http://www.planalto.gov.br/ccivil_03/leis/ leis_2001//10216.htm

Brasil. Ministério da Saúde. Secretaria de Assistência à Saúde. (2002). Notificação de maus-tratos contra crianças e adolescentes pelos profissionais de saúde: um passo a mais na cidadania em saúde. Brasília. Recuperado em 09 de setembro de 2013: http://bvsms.saude.gov. br/bvs/publicacoes/notificacao_maustratos_ criancas_adolescentes.pdf

Brasil, H. H. A. (2003). Desenvolvimento da versão brasileira da K-SADS-PL (Schedule for Affective Disorders and Schizophrenia for School Aged
Children Present and LifetimeVersion) e estudo de suas propriedades psicométricas. Tese de Doutorado, Curso de Pós-Graduação em Psiquiatria e Psicologia Médica, Universidade Federal de São Paulo, São Paulo, SP.

Brasil. (2004a). Resolução no 15, de 15 de outubro de 2004. Diário Oficial da União, Brasília, DF. Recuperado em 09 de setembro de 2013, de http://www.mds.gov.br/acesso-a-informacao/ legislacao/mds/resolucoes/2004/Resolucao $\% 20$ no\%20145-\%20de\%2015\%20de\%20outubro\%20 de\%202004.pdf/view

Brasil. Ministério da Saúde. (2004b). Saúde mental no SUS: os centros de atenção psicossocial. Recuperado em 02 de dezembro de 2013, de http://www.ccs.saude.gov.br/saude_mental/ pdf/SM_Sus.pdf

Brasil. Secretaria de Direitos Humanos. Programa Nacional de Enfrentamento da Violência Sexual Contra Crianças e Adolescentes. (2010). Relatório disque denúncia nacional. Brasília. Recuperado em 09 de setembro de 2013, de http://www.direitosdacrianca.org.br/midiateca/ publicacoes/relatorio-geral-do-disque-100-2010

Brasil. Ministério da Saúde. (2012). Caderno Saúde Mental em Dados 10, VII(10). Informativo eletrônico. Recuperado em 09 de setembro de 2013 de http://portal.saude.gov.br/portal/ arquivos/pdf/mentaldados10.pdf

Brasil. (2013). Lei no 12.845, de 1으 de agosto de 2013. Diário Oficial da União, Brasília, DF. Recuperado em 09 de setembro de 2013, de http://www.planalto.gov.br/ccivil 03/ ato20112014/2013/lei/l12845.htm

Cohen, J. A., Deblinger, E., Mannarino, A. P., \& Steer, R. A. (2004). A multisite, randomized controlled trial for children with sexual abuse-related PTSD symptoms. Journal of the American Academy of Child and Adolescent Psychiatry, 43(4), 393-402. http://dx.doi. org/10.1097/00004583-200404000-00005. PMid:15187799

Cohen, J. A., Mannarino, A. P., \& Knudsen, K. (2005). Treating sexually abused children: 1 year follow-up of a randomized controlled trial. Child Abuse \& Neglect, 29(2), 135-145. http:// dx.doi.org/10.1016/j.chiabu.2004.12.005. PMid:15734179

Conselho Federal de Psicologia - CFP. (2009). Serviço de proteção social a crianças e adolescentes vítimas de violência, abuso e exploração sexual 
e suas famílias: referências para a atuação do psicólogo. Brasília.

Conselho Federal de Psicologia - CFP. (2000). Resolução no 10, de 20 de dezembro de 2000. Recuperado em 09 de setembro de 2013: http:// site.cfp.org.br/wp-content/uploads/2000/12/ resolucao2000_10.pdf

Deslandes, S. F. (2013). Sumário de pesquisa: avaliação das estratégias governamentais municipais no enfrentamento da violência sexual e exploração sexual de crianças e adolescentes em quatro capitais brasileiras: Porto Alegre. Rio de Janeiro: Fiocruz.

Dube, S. R., Anda, R. F., Whitfield, C. L., Brown, D. W., Felitti, V. J., Dong, M., et al. (2005). Long-term consequences of childhood sexual abuse by gender of victim. American Journal of Preventive Medicine, 28(5), 430-438. http:// dx.doi.org/10.1016/j.amepre.2005.01.015. PMid:15894146

Freitas, C. P., \& Habigzang, L. H. (2014). Tecnologia social de capacitação profissional para intervenção com vítimas de violência sexual. In L. F. Habigzang, E. Diniz, \& S. H. Koller (Eds.), Trabalhando com adolescentes: teoria e intervenção psicológica (pp. 309-328). Porto Alegre: Artmed.

Gonçalves, H. S., \& Ferreira, A. L. (2002). A notificação da violência intrafamiliar contra crianças e adolescentes por profissionais de saúde. Cadernos de Saude Publica, 18(1), 315-319. http://dx.doi.org/10.1590/S0102311X2002000100032. PMid:11910450

Habigzang, L. F., Azevedo, G. A., Koller, S. H., \& Machado, P. X. (2006). Fatores de risco e de proteção na rede de atendimento a crianças e adolescentes vítimas de violência sexual. Psicologia: Reflexão e Crítica, 19(3), 379-386. http://dx.doi.org/10.1590/S010279722006000300006.

Habigzang, L. F., Stroeher, F. H., Hatzenberger, R., Cunha, R. C., Ramos, M. S., \& Koller, S. H. (2009). Grupoterapia cognitivo-comportamental para crianças e adolescentes vítimas de abuso sexual. Revista de Saúde Pública, 43(1), 70-78. PMid:19169577.

Habigzang, L. F., Ramos, M. S., \& Koller, S. H. (2011). A revelação de abuso sexual: Medidas adotadas pela rede de apoio. Psicologia: Teoria e Pesquisa (Brasília), 27(4), 467-473. http://dx.doi. org/10.1590/S0102-37722011000400010.
Habigzang, L. F., Damásio, B. F., \& Koller, S. H. (2013). Impact evaluation of a cognitive behavioral group therapy model in Brazilian sexually abused girls. Journal of Child Sexual Abuse, 22(2), 173-190. http://dx.doi.org/10.10 80/10538712.2013.737445. PMid:23428150

Hetzel-Riggin, M. D., Brausch, A. M., \& Montgomery, B. S. (2007). A meta-analytic investigation of therapy modality outcomes for sexually abused children and adolescents: an exploratory study. Child Abuse \& Neglect, 31(2), 125-141. http:// dx.doi.org/10.1016/j.chiabu.2006.10.007. PMid:17306369

Hillberg, T., Hamilton-Giachritsis, C., \& Dixon, L. (2011). Review of meta-analyses on the association between child sexual abuse and adult mental health difficulties: a systematic approach. Trauma, Violence \& Abuse, 12(1), 38-49. http://dx.doi.org/10.1177/1524838010386812. PMid:21288934

Hohendorff, J. V., \& Habigzang, L. F. (2014). Atuação do profissional da psicologia na avaliação e intervenção em situações de violência sexual contra adolescentes. In S. H. Koller, E. Diniz, \& L. F. Habigzang (Eds.), trabalhando com adolescentes: uma perspectiva bioecológica e aplicada (pp. 293-308). Porto Alegre: Artmed.

Hohendorff, J. V., Salvador-Silva, R., Andrade, R., Habigzang, L. F., \& Koller, S. H. (2014). Adaptação e avaliação de uma intervenção cognitivo-comportamental para meninos vítimas de violência sexual. Psicologia: Reflexão e Crítica, 27(3), 424-433.

Kaufman, J., Birmaher, B., Brent, D., Rao, U., Flynn, C., Moreci, P., et al (1997). Schedule for affective disorders and schizophrenia for school-age children-present and lifetime version (K-SADS-PL): initial reliability and validity data. Journal of the American Academy of Child and Adolescent Psychiatry, 36(7), 980-988. http:// dx.doi.org/10.1097/00004583-19970700000021. PMid:9204677

Lucânia, E. L., Valério, N. I., Barison, S. Z. P., \& Miyazaki, M. C. O. S. (2009). Intervenção cognitivo-comportamental em violência sexual: estudo de caso. Psicologia em Estudo, 14(4), 817-826. http://dx.doi.org/10.1590/S141373722009000400022 .

Pelisoli, C., Pires, J. P. M., Almeida, M. E., \& Dell'Aglio, D. D. (2010). Violência sexual contra crianças e adolescentes: Dados de um serviço de referência. Temas em Psicologia, 18(1), 85-97. 
Pereda, N., Guilera, G., Forns, M., \& Gómez-Benito, J. (2009). The prevalence of child sexual abuse in community and student samples: a meta-analysis. Clinical Psychology Review, 29(4), 328-338. http://dx.doi.org/10.1016/j. cpr.2009.02.007. PMid:19371992

Romano, E., \& De Luca, R. V. (2001). Male sexual abuse: a review of effects, abuse characteristics, and links with later psychological functioning. Aggression and Violent Behavior, 6(1), 55-78. http:// dx.doi.org/10.1016/S1359-1789(99)00011-7.

Williams, L. C. A. (2002). Abuso sexual infantil. In H. J. Guilhardi, M. B. B. Madi, P. P. Queiroz, \& M. C. Scoz (Eds.), Sobre comportamento e cognição: contribuições para a construção da teoria do comportamento (Vol. 10, pp. 155-164). Santo André: ESETec.

World Health Organization - WHO. (1999). WHO recognizes child abuse as a major public health problem. Recuperado de http://www.who.int/ inf-pr-1999/en/pr99-20.html

World Health Organization - WHO \& International Society for Prevention of Child Abuse and Neglect - ISPCAN (2006). Preventing child maltreatment: a guide to taking action and generating evidence. Suíça. Recuperado de http://whqlibdoc.who. int/publications/2006/9241594365_eng.pdf 